\title{
Ultrasonographic Bone Evaluation in Young Women with Type 1 Diabetes
}

\author{
Borzì G ${ }^{1}$, Russo $M^{2}$, Guardo $F^{1}$, Migliorino $V^{1}$, Frittitta $L^{1}$, Sciacca $L^{1}$ and Padova $G^{3}$ \\ ${ }^{1}$ Endocrinology, Department of Clinical and Experimental Medicina, University of Catania, Garibaldi-Nesima Hospital, \\ Catania, Italy
}

${ }^{2}$ Endocrinology, Department of Clinical and Experimental Medicine, University of Messina, Messina, Italy

${ }^{3}$ Endocrinology Unit, Garibaldi-Nesima Hospital, Catania, Italy

Received: March 09, 2018; Accepted: April 04, 2018; Published: April 11, 2018

*Corresponding author: Borzì G, U.O. Endocrinologia,Ospedale Garibaldi Nesima, Via Palermo 636, 95122 Catania, Italy; Fax: +39-095472988; E-mail: graziella.borzi@yahoo.it

\begin{abstract}
Purpose: A relationship between Type 1 Diabetes Mellitus (T1DM) and an impaired bone quality is well known, although the pathogenesis of this link is not yet clear. In this study we have investigated the bone status in young women with T1DM, using the Bone Quantitative Ultra-Sound (bQUS) at the hand phalanges, a screening tool of osteoporosis. We preferred bQus to DXA because of the young age of the population recruited and the easy accessibility of the device, although, recently, the absence of technology-specific guidelines has created uncertainty respect to the application of bQUS results in the management of individual patients in clinical practice. However, in past, studies in vitro showed that bQUS parameters were significantly associated with Bone Mineral Density (BMD), bone micro architecture and mechanical indices. In humans, bQUSparameters were found to be associated with BMD as well, then this tool could discriminate and screen subjects and predict risk for future fracture.

Methods: A consecutive series of 55 young women affected by T1DM were recruited and subjected to phalangeal bQUS. The glycemic control, estimated by HbA1c values, insulin requirement, major risk factors for osteoporosis, chronic diabetic complications and associated autoimmune diseases were evaluated. Bone-QUS data obtained were compared with those of a group of matched healthy controls.

Results: The prevalence of osteoporosis in patients group is very small; however T1DM women showed worse ultrasonographic bone parameters than healthy controls. Their data did not correlate to the age of patients, Body Mass Index (BMI), diabetes onset and duration of the disease and not differ in relation to the presence of chronic diabetic complications and autoimmune diseases, kind of insulin administration and cigarette smoking. Instead, significant correlations between ultrasonographic values and HbA1c (negative correlation, $\mathrm{p}<0,05$ ), insulin daily requirement (positive correlation, $\mathrm{p}<0,02$ ) and calcium dietary intake (positive correlation $\mathrm{p}<0,02$ ) were found.
\end{abstract}

Conclusion: This study confirms the bone status alterations in T1DM, even in young women compared with healthy controls. The bone quality is related to the glycemic control and insulin requirement, but not to the diabetes duration. Moreover, a low calcium dietary intake could worsen the bone health status. Then, strategies to prevent and treat osteoporosis in people with diabetes should be applied: a diet rich in calcium, vitamin D supplementation, exercise, healthy lifestyle, bone density test and an effective glycemic control should be encouraged in order to prevent diabetesinduced osteoporosis.

The bQUS is a method easily accessible, low-cost, non-invasive, non-ionizing, that could be widely used for osteoporosis screening in young patients with T1DM.

Keywords: mellitus diabetes; osteoporosis; phalangeal ultrasonography; bone; women.

\section{Introduction}

A relationship between Type 1 Diabetes (T1DM) and an increased fracture risk is known.

An inadequate bone mass peak achievement in children with pre pubertal onset diabetes has been proposed as a major factor contributing to reduced bone quality and osteoporosis in later life. In T1DM it was also been reported a negative calcium balance as a result of hypercalciuria during periods of hyperglycemia, functional hypoparathyroidism, vitamin D deficiency and alterations in vitamin D metabolism [1].

Many studies showed that a poor glycemic control in patients with T1DM is associated with osteopaenia and osteoporosis by pro inflammatory cytokines like TNF $\alpha$, which inhibits osteoblast differentiation and activity, thus increasing osteoblast apoptosis and by reactive oxygen species, which in turn can increase osteoclast formation and activity [2-6].

The insulin anabolic effects on bone, often mediated by Insulin-like Growth Factor-1 (IGF-1), are known [7]. In patients with uncontrolled T1DM, the levels of free IGF-1 are low due to increase of IGF-binding proteins, particularly IGFBP3 [8]. Furthermore, an intensive insulin therapy has been shown to stabilize the Bone Mineral Density (BMD) in patients with T1DM [9]. 
The aim of this study was to analyze the bone status of a group of young women with T1DM, by using an easily accessible method, the Bone Quantitative Ultra-Sound (bQUS) at the hand phalanges. The gold standard method recommended by the World Health Organization for the diagnosis of osteoporosis is dual-Xray absorptiometry (DXA); using this method, osteoporosis is defined by a BMD lower than -2.5 standard deviations (SD) of the refer-ence BMD of Caucasian women aged 20-29 years. The b-QUS is a method widely used, low-cost, non-invasive, nonionizing and obtained by a portable ultrasound and it could be used when DXA is not available and/or when we want to avoid ionizing radiation, such as in young patients. It uses the speed of sound (SOS) as a principle to assess bone mass in the proximal phalanges of the hand, a site sensitive to bone alterations and less influenced by bone size [10-11].

Previous in vitro studies examining the relationship between bQUS and bone properties found that SOS was closely related to BMD [12-16]. Toyras et al. indicated that this relationship was strong, with a coefficient of correlation (r) of 0.888 [17]. Significant correlations between SOS with microarchitecture indices of the bone were also discovered. In humans, there were significant correlations between bQUS indices and BMD values at various body sites assessed cross-sectionally. The study of Varenna et al. in 4832 Italian men aged 60-80 years found that bQUS indices were significantly associated with history of hip fracture and non-spinal fracture [18]. These observations from cross-sectional studies were further validated by prospective studies. In the Norfolk Cohort Study involving 14824 men and women aged 42-82 years followed for 1.9 years, Khaw et al discovered that one SD decrease in ultrasound velocity translated to a $60 \%$ increase in fracture risk in both genders [19].

\section{Materials and Methods}

Fifty-five pre-menopause women with T1DM, followed at the Diabetes Center of Garibaldi Nesima Hospital in Catania, were recruited.

The osteoporosis major risk factors were investigated: cigarette smoking, deficient calcium diet, familiar history of osteoporosis and/or osteoporotic fractures, personal history of atraumatic fractures, gastro-intestinal diseases associated with malabsorption, therapies influencing bone density, long periods of amenorrhea (> 6 months) and premature ovarian failure $(<$ 40 years). In particular, the calcium dietary intake was estimated using a food diary. The patients had to write down each food and beverage at the time it was consumed; we asked them not to alter their usual eating habits. Calcium contents of the foods recorded were analyzed using a nutritional assessment software program. Food diaries are often used as gold standards for evaluation of nutrients intake, also in estimation of calcium dietary intake [2024].

All patients (pts) were subjected to the macro-angiopathic and micro-angiopathic chronic complications screening by epiaortic trunks and lower limb arteries echo-doppler, electrocardiography, microalbuminuria test, fundus oculi examination and clinical evaluation of peripheral and autonomic neuropathy by monofilament test, biothesiometry and cardiovascular autonomic function tests.

Chronic thyroiditis, primitive hypoadrenalism and celiac disease were also investigated.

Medium hba1c (hplc method) and medium total daily insulin requirements (units $/ \mathrm{kg} / \mathrm{die}$ ) of the last five years and the last year were calculated, in order to estimate the glycemic control and insulin needs.

Phalangeal bQUS (DBM Sonic Bone Profiler - IGEA) was performed in all pts. A specific software automatically calculated the Amplitude Dependent Speed of Sound (Ad-Sos), expressed in $\mathrm{m} / \mathrm{s}$, and $\mathrm{t}$-score and $\mathrm{z}$-score values. Values of $\mathrm{t}$-score $<-3.2$ and between - 3.2 and - 1 ds were used for diagnosis of osteoporosis and osteopaenia respectively [10].

Thirty-six non-diabetic young women, selected among medical and paramedical staff of the hospital and matched for age, BMI, smoking habits, calcium intake and familiar history of osteoporosis and without the major risk factors for osteoporosis, were used as controls.

The statistical analysis was performed by the software Statview. Descriptive data were expressed as mean \pm sd. Fisher's test was used to compare categorical variables. Student's t test for unpaired data was used to compare data with parametric distribution. Data with non-parametric distribution and differences between groups were analyzed with the MannWhitney U-test. Correlations between variables were evaluated by calculating the spearman's coefficient.

\section{Results}

The mean age of the pts was 32 yrs ( $S D \pm 8.16)$; the mean diabetes duration was 18.2 yrs (SD \pm 8.8 ) and in 23 pts it was above 20 yrs; 32 pts had a pre- or peri-puberal diabetes onset; the mean Body Mass Index (BMI) was $23.6 \mathrm{~kg} / \mathrm{mq}$ (SD \pm 3.5 ) [Table 1].

The major risk factors for osteoporosis in pts group were present as follows: 7 smokers, 7 pts with low calcium intake ( $<1200 \mathrm{mg} /$ die), 11 with familiar history of osteoporosis and/ or osteoporotic fractures, 2 with celiac disease (under glutenfree diet). None of the pts practiced therapies that influence bone density or interfere with calcium-phosphorus metabolism; none referred long periods of amenorrhea or suffered from a premature ovarian failure. In control group there are: 5 smokers, 5 with low calcium intake $(<1200 \mathrm{mg} / \mathrm{die}), 8$ with familiar history of osteoporosis and/or osteoporotic fractures; all above mentioned osteoporosis risk factors were absent (any significant difference with the patients group).

The chronic complications screening in patients group showed: 9 pts with peripheral atheromasia without significant hemodynamic vascular stenosis; 28 with microangiopathy complications: 10 with initial diabetic retinopathy, 10 with positive microalbuminuria test, defined as albumin excretion of 30-299 mg/24 hours, and 8 with both factors. Major chronic complications (peripheral atheromasia with vascular stenosis, 
Table 1: Clinical characteristis of the women with T1DM and health controls recruited

\begin{tabular}{|c|c|c|c|}
\hline Clinical characteristics & women with T1DM n = 55 Mean (SD) & $\begin{array}{l}\text { Health women } \mathrm{n}=35 \\
\quad \text { Mean (SD) }\end{array}$ & $\mathbf{p}$ \\
\hline Age (years) & $32.0(8.6)$ & $31.7(8.0)$ & NS \\
\hline Diabetes duration (years) & $18.2(8.8)$ & - & \\
\hline BMI (Kg/mq) & $23.6(3.5)$ & $23.1(5.6)$ & NS \\
\hline HbA1c of the last five years $(\mathrm{mmol} / \mathrm{mol})$ & $61.0(24.0)$ & - & \\
\hline Hba1c of the last year ( $\mathrm{mmol} / \mathrm{mol})$ & $55.0(13.1)$ & - & \\
\hline $\begin{array}{l}\text { Daily insulin requirement of the last five } \\
\text { years (UI/ } \mathrm{kg} / \mathrm{die})\end{array}$ & $0.7(0.2)$ & - & \\
\hline $\begin{array}{l}\text { Daily insulin requirement of the last year } \\
\qquad(\mathrm{UI} / \mathrm{kg} / \mathrm{die})\end{array}$ & $0.7(0.3)$ & - & \\
\hline Frequency of osteoporosis risk factors & $\begin{array}{l}\text { women with T1DM n = } 55 \\
\text { n. (\%) }\end{array}$ & $\begin{array}{l}\text { Health women } \mathrm{n}=35 \\
\text { n. (\%) }\end{array}$ & $\mathbf{p}$ \\
\hline Smokers & $7(12.7 \%)$ & $5(13.8)$ & NS \\
\hline Calcium dietary intake $<1200 \mathrm{mg} /$ die & $7(12.7 \%)$ & $5(13.8)$ & NS \\
\hline Familiar history of osteoporosis & $11(20.0 \%)$ & $8(22.2 \%)$ & NS \\
\hline Personal history of atraumatic fractures & - & - & NS \\
\hline Celiac disease & $2(3.6 \%)$ & - & NS \\
\hline Therapy influencing bone metabolism & - & - & NS \\
\hline Amenorrhea $>6$ months & - & - & NS \\
\hline Chronic diabetes complications & Women with T1DM n = 55 n. (\%) & & \\
\hline Peripheral atheromasia & $9(16.4 \%)$ & & \\
\hline Initial diabetic retinopathy & $18(32.7 \%)$ & & \\
\hline Positive microalbuminuria & $18(32.7 \%)$ & & \\
\hline Multiple daily injection & $35(63 \%)$ & & \\
\hline Continuous subutaneous insulin infusion & $20(37 \%)$ & & \\
\hline
\end{tabular}

signs of ischemic cardiomyopathy and cerebropathy, advanced and/or laser-treated diabetic retinopathy and diabetic nephropathy) were absent, so also signs of diabetic neuropathy. In addition, 24 pts had at least one autoimmune disease (chronic thyroiditis in euthyroidism, celiac disease under gluten-free diet and multiple sclerosis in pharmacological therapy).

Most of the pts practiced multi-injective insulin therapy (multiple daily injection, MDI), while 20 of them practiced continuous subcutaneous insulin infusion (CSII) by pump.

The mean hba1c of the last five yrs amounted to $7.7 \%$ (SD $\pm 2.6)-61 \mathrm{mmol} / \mathrm{mol}(\mathrm{SD} \pm 24.0)$, while the mean value of the last year was $7.2 \%(S D \pm 2.6)-55 \mathrm{mmol} / \mathrm{mol}(\mathrm{SD} \pm 13.1)$.

The mean total daily insulin requirement of the last five yrs amounted to $0.7 \mathrm{UI} / \mathrm{Kg} /$ die $(\mathrm{SD} \pm 0.2$ ) and the last year was 0.7 $\mathrm{UI} / \mathrm{kg} /$ die $(\mathrm{SD} \pm 0.3)$.

Using bQUS we found: only 1 patient with osteoporosis (1.8\%), 11 pts with osteopaenia (20\%) and 43 pts with normal estimated bone density (78.2\%).
The pts age, diabetes duration and BMI failed to show a significant correlation with the AD-Sos [Figure 1]. Moreover, we did not found any significant difference in ultrasonographic data in relation to pre- or post- pubertal diabetes onset. A significant negative correlation between AD-Sos and hba1c of the last 5 years and a significant positive correlation between the ultrasonographic value and the daily insulin requirement in the past 5 years and in the current year were observed [Figure 2].

It was not a statistically significant difference in bQUS parameters between smokers and non-smokers, MDI and CSII. Instead, significant lower ultrasonographic data in patients with low calcium diet were reported $(\mathrm{p}<0.05)$ [Figure 3].

The control group, matched for age (31.7 $\pm 8.0 \mathrm{yrs})$, BMI $(23.1 \pm 5.6 \mathrm{~kg} / \mathrm{mq})$, smoking habits, calcium intake and familiar history of osteoporosis and without the major risk factors for osteoporosis, showed statistically higher AD-Sos values versus the pts $(\mathrm{p}<0.05, \mathrm{AD}-\mathrm{sos} 2130.9 \pm 83.5 \mathrm{~m} / \mathrm{s}$ and $2083.7 \pm 68.3$, respectively). 
A

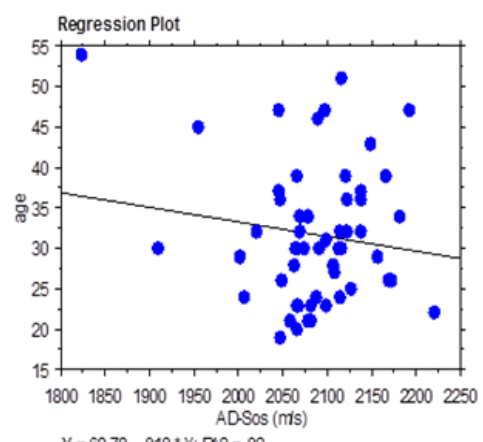

\section{B}

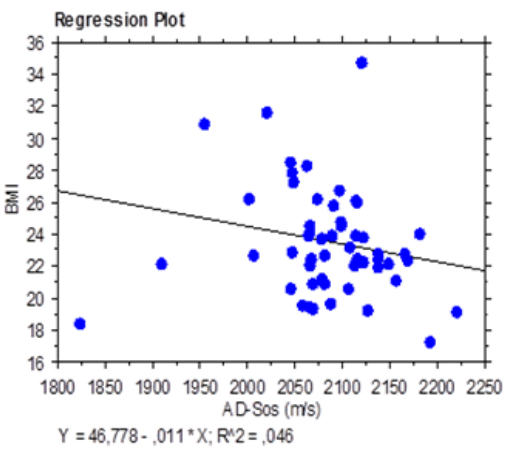

C

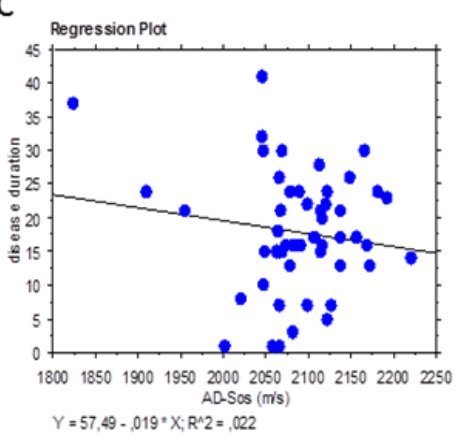

Figure 1: Absence of correlation between AD-Sos and: A) age, B) diabetes duration, C) BMI.

A) POSITIVE CORRELATION BETWEEN DAILY INSULIN REQUIREMENT IN THE LAST 5 YEARS AND AD-Sos $(p<0,02)$
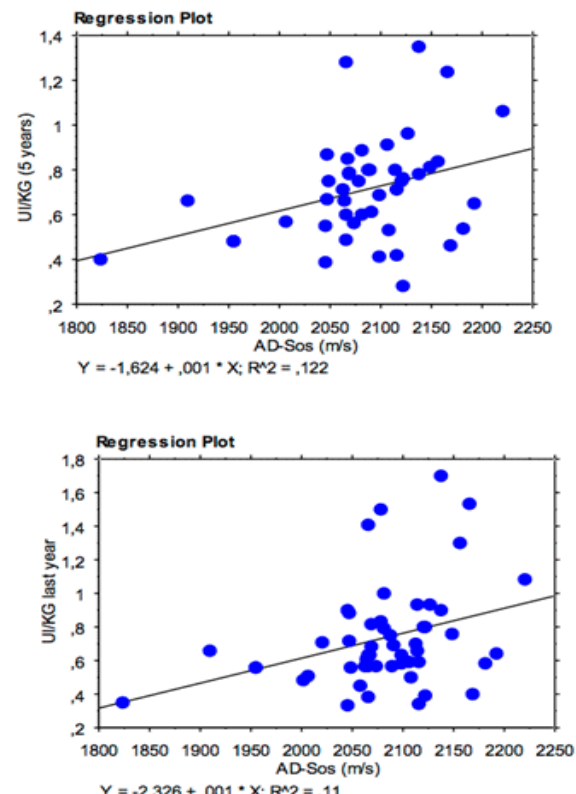

Figure 2: A correlation between A) daily insulin requirement in the last 5 years and AD-Sos ( $<<0.02)$, B) daily insulin requirement in the last year and AD-Sos ( $<<0.02), C)$ medium HbA1c in the last 5 years and AD-Sos $(p<0.05)$, VALUES IN THE LAST FIVE YEARS AND AD-SOS $(p<0,05)$

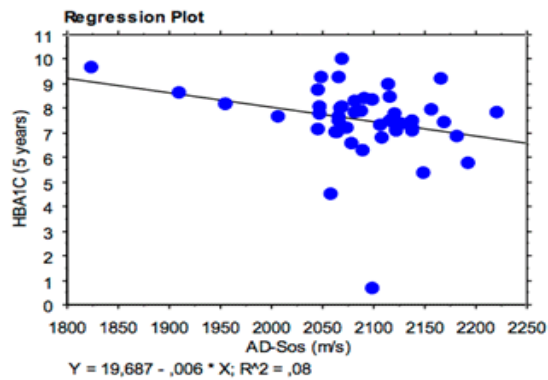

B) Positive correlation between daily insulin requirement in the last year and AD-Sos $(p<0,05)$
C) NEGATIVE CORRELATION BETWEEN HBA1C 


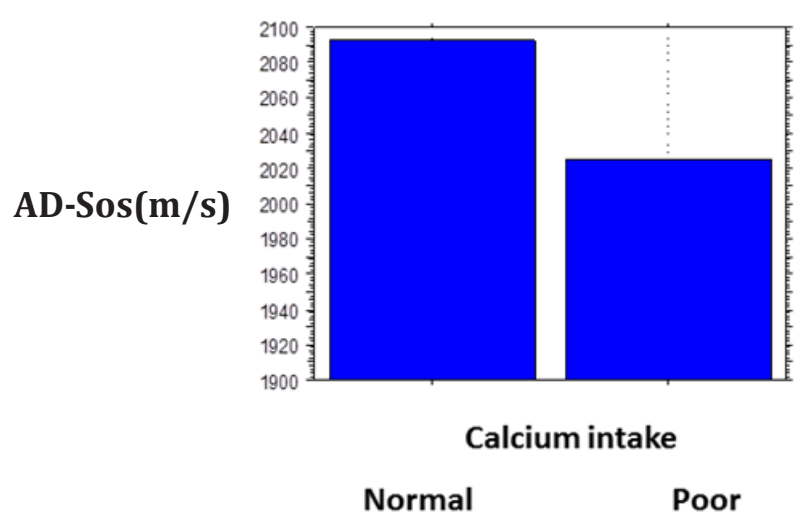

Figure 3: Significant lower AD-Sos in patients with low calcium diet.

\section{Discussion}

The correlation between bone loss and diabetes is particularly complex and not yet clear. If the link between type 2 diabetes and osteoporosis appears to be controversial and debated, many studies documented a reduction of bone mineral density and quality in T1DM [2, 25-29].

The attainment of bone mass peak may be affected by the early disease onset, resulting in the failure to achieve an adequate bone density [30]. In our study, We did not find a statistically significant correlation between $\mathrm{AD}-\mathrm{SoS}$ and both age and disease duration of T1DM. In fact, according to some studies [31-32], in young patients and/or with a few years of the disease, the bone quality may already be compromised.

A significant negative correlation between all the ultrasonographic parameters and glycemic control was found: higher mean HbA1c values, expression of a poor glycemic control, are associated with lower densitometric parameters (AD-SoS, T-score and Z-score) with evidence of an alteration of bone status.

The degree of glycemic control could have a considerable influence in the skeletal health. In T1DM animal models has been shown that poor glycemic control leads to the alteration of bone mineralization, probably due to altered expression of genes encoding pro-osteoblastic proteins, impaired ability of bone mineralization, decreased osteoblast and osteoclast activity, with a general decrease in bone remodeling [33].

A significant positive correlation between the densitometric parameters and insulin requirement was found. Moreover, insulin needs did not relate to the duration of the diabetes in the examined patients. This result could be an expression of the anabolic insulin action at the bone level. In animal models it has also been shown that insulin treatment may improve the bone quality and strength [33].

The type of insulin administration (MIIT vs. CSSI) does not seem to influence our results.

Instead, poor calcium dietary intake significantly affected lower densitometric parameters. It is known the importance of calcium in bone health, from the first months of life up to the bone mass peak [34]. A proper calcium intake can be obtained by milk and dairy products, highly mineral waters and calcium enriched foods.

In addition to a general low calcium intake in the population, gastrointestinal diseases such as celiac disease can prevent proper absorption of calcium.

The effect of cigarette smoking on bone is known. In our study, there was not a statistically significant correlation between smoking and low density values, probably due to the little sample and the young age of the patients [35].

\section{Conclusion}

Although the small sample size of patients and controls is a crucial limit of the study, our data confirmed that type 1 diabetes has been long associated with alterations in bone quality, and diabetic women of childbearing age have significantly lower AD-Sos values than non-diabetics. Phalangeal bone ultra-sound has proved to be a valuable diagnostic tool, given the low cost and the absence of risk, to perform an adequate screening of osteoporosis, above all in younger patients, thus implementing a long-term monitoring and proper prevention of possible fractures [25-26]. Above the possible early bone alterations linked to etiopathogenetic mechanism in $\mathrm{t} 1 \mathrm{dm}$, the good glycemic control and not the disease duration seems to be the most important determinant of bone quality in diabetic patients, together with an adequate calcium intake.

Osteoporosis, as other diabetic complications, should be screened and prevented in order to reduce the future fracture risk.

\section{Declaration}

\section{Conflict of Interest}

The authors declare that they have no conflict of interest.

\section{Ethical Approval}

All procedures performed during the study were in accordance with the clinical standards of institutional and/or national research committee and the 1964 Helsinki declaration and its later amendments or comparable ethical standards.

\section{Informed Consent} study.

All patients provided informed consent to participate in the

\section{References}

1. Thalassinos NC, Hadjiyanni P, Tzanela M, Alevizaki C, Philokiprou D. Calcium metabolism in diabetes mellitus: Effect of improved blood glucose control. Diabet Med. 1993;10(4):341-344.

2. Vestergaard P. Discrepancies in bone mineral density and fracture risk in patients with type 1 and type 2 diabetes: A meta-analysis. Osteoporos Int. 2007;18(4):427-444. Doi: 10.1007/s00198-006-0253-4

3. Joshi A, Varthakavi P, Chadha M, Bhagwat N. A study of bone mineral density and its determinants in type 1 diabetes. J Osteoporos. 2013;2013:397814.50. Doi: 10.1155/2013/397814 
4. Lechleitner M, Koch T, Herold M, Dzien A, Hoppichler F. Tumour necrosis factor-alpha plasma level in patients with type 1 diabetes mellitus and its association with glycaemic control and cardiovascular risk. J Intern Med. 2000;248(1):67-76.

5. Coe LM, Irwin R, Lippner D, McCabe LR. The bone marrow microenvironment contributes to type I diabetes induced osteoblast death. J Cell Physiol. 2011;226(2):477-483. Doi: 10.1002/jcp.22357

6. Fraser JH, Helfrich MH, Wallace HM, Ralston SH. Hydrogen peroxide, but not superoxide, stimulates bone resorption in mouse calvariae. Bone. 1996;19(3):223-226.

7. Nixon AJ, Lillich JT, Burton-Wurster N, Lust G, Mohammad HO Differentiated cellular function in fetal chondrocytes cultured with insulin-like growth factor-I and transforming growth factor-beta. J Orthop Res. 1998;16(5):531-541. Doi: 10.1002/jor.1100160503

8. Moyer-Mileur LJ, Slater H, Jordan KC, Murray MA. IGF-1 and IGFbinding proteins and bone mass, geometry, and strength: Relation to metabolic control in adolescent girls with type 1 diabetes. J Bone Miner Res. 2008;23(12):188-191. Doi: 10.1359/jbmr.080713

9. Campos Pastor MM, López-Ibarra PJ, Escobar-Jiménez F, Serrano Pardo MD, García-Cervigón AG. Intensive insulin therapy and bone mineral density in type 1 diabetes mellitus: A prospective study. Osteoporos Int 200;11(5):455-459.

10.C. Wüster, Albanese C, de Aloysio D, Duboeuf F, Gambacciani M Gonnelli S, et al. Phalangeal osteosonogrammetry study (PhOS): age related changes, diagnostic sensitivity and discrimination power Journal of Bone and Mineral Research. 2000;15(8):1603-1614. Doi: 10.1359/jbmr.2000.15.8.1603

11.Guglielmi G, Cammisa M, De Serio A, Scillitani A, Chiodini I, Carnevale $\mathrm{V}$, et al. Phalangeal US velocity discriminates between normal and vertebrally fractured subjects. European Radiology. 1999;9(8):16321637.

12.Cavani F, Giavaresi G, Fini M, Bertoni L, de Terlizzi F, Barkmann R. et al. Influence of density, elasticity, and structure on ultrasound transmission through trabecular bone cylinders. IEEE Trans Ultrason Ferroelectr Freq Control. 2008;55(7):1465-1472. Doi: 10.1109/ TUFFC.2008.821

13.Cortet B, Boutry N, Dubois P, Legroux-Gérot I, Cotten A, Marchandise X. Does quantitative ultrasound of bone reflect more bone mineral density than bone microarchitecture? Calcif Tissue Int. 2004;74(1):60-67.

14.Hans D, Wu C, Njeh CF, Zhao S, Augat P, Newitt D. et al. Ultrasound Velocity of Trabecular Cubes Reflects Mainly Bone Density and Elasticity. Calcif Tissue Int. 1999;64(1):18-23.

15.Padilla F, Jenson F, Bousson V, Peyrin F, Laugier P. Relationships of trabecular bone structure with quantitative ultrasound parameters: In vitro study on human proximal femur using transmission and backscatter measurements. Bone. 2008;42(6):1193-1202. Doi: 10.1016/j.bone.2007.10.024

16.Töyräs J, Kröger H, Jurvelin JS. Bone properties as estimated by mineral density, ultrasound attenuation, and velocity. Bone. 1999;25(6):725731.

17.Töyräs J, Nieminen MT, Kröger H, Jurvelin JS. Bone mineral density, ultrasound velocity, and broadband attenuation predict mechanical properties of trabecular bone differently. Bone. 2002;31(4):503-507.

18.Varenna M, Sinigaglia L, Adami S, Giannini S, Isaia G, Maggi S. et al. Association of quantitative heel ultrasound with history of osteoporotic fractures in elderly men: the ESOPO study. Osteoporos Int. 2005;16(12):1749-54. Doi: 10.1007/s00198-005-1914-4
19.Khaw KT, Reeve J, Luben R, Bingham S, Welch A, Wareham N. et al. Prediction of total and hip fracture risk in men and women by quantitative ultrasound of the calcaneus: EPIC-Norfolk prospective population study. Lancet. 2004;363(904):197-202. Doi: 10.1016/ S0140-6736(03)15325-1

20.Pietinen P, Hartman AM, Haapa E, et al. Reproducibility and validity of dietary assessment instruments. I. A self-administered food use questionnaire with a portion size picture booklet. Am J Epidemiol. 1988;128(3):655-666.

21.Pietinen P, Hartman AM, Haapa E, et al. Reproducibility and validity of dietary assessment instruments. II. A qualitative food frequency questionnaire. Am J Epidemiol. 1988;128(3):667-676

22.Xu L, Porteous JE, Phillips MR, et al. Development and validation of a calcium intake questionnaire for postmenopausal women in China. Ann Epidemiol. 2000;10(3):169-75.

23.Brown JL, Griebler R. Reliability of a short and long version of the Block food frequency form for assessing changes in calcium intake. J Am Diet Assoc. 1993;93(7):784-792.

24.Block G, Hartman AM, Naughton D. A reduced dietary questionnaire: development and validation. Epidemiology. 1990;1(1):58-64.

25.Heap J, Murray MA, Miller SC, Jalili T, Moyer-Mileur LJ. Alterations in bone characteristics associated with glycemic control in adolescents with type 1 diabetes mellitus. J Pediatr. 2004;144(1):56-62.

26.Liu EY, Wactawski-Wende J, Donahue RP, Dmochowski J, Hovey KM, Quattrin T. Does low bone mineral density start in post teenage years in women with type 1 diabetes? Diabetes Care. 2003;26(8):2365-2369.

27.Buysschaert M, Cauwe F, Jamart J, Brichant C, De Coster O, Magnan A, et. al. Proximal femur density in type 1 and 2 diabetic patients. Diabetes Metab. 1992;18(1):32-37.

28.Compston JE, Smith EM, Matthews C, Schofield P. Whole body composition and regional bone mass in women with insulin dependent diabetes mellitus. Clin Endocrinol. 1994;41(3):289-293.

29.Forst T, Pfutzner A, Kann P, Schehler B, Lobmann R, Schafer H, et. al. Peripheral osteopenia in adult patients with insulin dependentdiabetes mellitus. Diab Med. 1995;12(10):874-879.

30.Mastrandrea LD, Wactawski-Wende J, Donahue RP, Hovey KM, Clark A, Quattrin T. Young women with type 1 diabetes have lower bone mineral density that persists over time, Diabetes Care 2008;31(9):1729-1735. Doi: $10.2337 / \mathrm{dc} 07-2426$

31.V. Carnevale, E. Romagnoli, E. D'Erasmo. Skeletal involvementin patients with diabetes mellitus. Diabetes Metab Res Rev. 2004;20(3):196-204. Doi: $10.1002 / d m r r .449$

32.Karagüzel G, Akçurin S, Ozdem S, Boz A, Bircan I. Bone mineral density and alterations of bone metabolism in children and adolescents with type 1 diabetes mellitus. J Pediatr Endocrinol Metab. 2006;19(6):805814. Doi: 10.1515/JPEM.2006.19.6.805

33.Thrailkill KM, Lumpkin CK Jr, Bunn RC, Kemp SF, Fowlkes JL. Is insulin an anabolic agent in bone? Dissecting the diabetic bone for clues. American Journal of Physiology - Endocrinology And Metabolism. 2005;289(5):E735-E745. Doi: 10.1152/ajpendo.00159.2005

34.Tai V, Leung W, Grey A, Reid IR, Bolland MJ. Calcium Intake and Bone Mineral Density: Systematic Review and Meta-analysis. BMJ 2015;351:h418. Doi: 10.1136/bmj.h4183

35.Ward KD, Klesges RC. A Meta-Analysis of the Effects of Cigarette Smoking on BoneMineral Density. Calcif Tissue Int. 2001;68(5):259270 . 